\title{
Wait-free Consensus in "In-phase" Multiprocessor Systems
}

\author{
Marina Papatriantafilou \\ Max-Planck-Institut für Informatik \\ Im Stadtwald, 66123 Saarbrücken, Germany \\ \& CTI \& Patras University, Greece \\ ptrianta@mpi-sb.mpg.de \\ Philippas Tsigas \\ Max-Planck-Institut für Informatik \\ Im Stadtwald, 66123 Saarbrücken, Germany \\ tsigas@mpi-sb.mpg.de
}

\begin{abstract}
In the consensus problem in a system with $n$ processes, each process starts with a private input value and runs until it chooses irrevocably a decision value, which was the input value of some process of the system; moreover, all processes have to decide on the same value. This work deals with the problem of wait-free-fully resilient to processor crash and napping failures-consensus of $n$ processes in an "in-phase" multiprocessor system. It proves the existence of a solution to the problem in this system by presenting a protocol which ensures that a process will reach decision within at most $n(n-3) / 2+3$ steps of its own in the worst case, or within $n$ steps if no process fails.

AMS Subject Classification (1991): 68M07, 68Q22, 68Q25, 90B12

CR Subject Classification (1991): B.3.2, B.4.3, B.4.5, C.1.2, C.2.4, C.4, D.1.3, D.4.1, D.4.5 Keywords \&S Phrases: Agreement, Consensus, Distributed Computation, Fault-Tolerance, Multiprocessor Systems, Napping Failures, Processor Crahses, Shared Memory, Wait-Free Synchronization.

Note: Partially supported by the EC ESPRIT II BRA ALCOM II (contr. \# 7141).

This work will also appear in the Proceedings of the 7th IEEE Symposium on Parallel and Distributed Processing
\end{abstract}




\section{Introduction}

In the consensus problem in a system with $n$ processes, each process starts with a private input value and runs until it chooses irrevocably a decision value, which has to be valid, i.e. to equal the input value of some process and consistent, i.e. it has to be the same value for all processes. Whereas this is no problem in an ideal, failure-free environment, it imposes certain constraints on the capabilities of an actual system, which is viable only if it permits protocols tolerant to failures. In a system with failures the consensus problem becomes a central issue of multiprocessor synchronization and coordination. Solutions which guarantee that each process decides after a certain number of its own steps, regardless of the other processes' relative speeds, are called wait-free. Wait-freedom is a desirable property in concurrent systems, since it helps in taking advantage of the inherent parallelism in the system by ensuring that no process may be blocked by others which might be slow, preempted, swapped out, delayed without warning by interrupts; moreover, it implies maximum tolerance to processor crash and napping failures.

As expected, such a fundamental problem received the attention of many researchers; as a result, many faces of the problem have been studied. In [12] and [20] it has been proven that in completely asynchronous systems -message passing and shared memory, respectively- not even one processor crash can be tolerated by a deterministic consensus protocol. In [9] the result is generalized for message passing systems; several critical parameters are identified and it is examined how they affect the number of faults that can be tolerated by a consensus protocol. In [14] shared memory data objects are partially classified according to the number of processes that can reach consensus in a wait-free manner using them. Recently, three groups with [7], [17] and [22], concurrently and independently have proven a conjecture first stated in [8], that even in the case when the agreement condition is weakened so that the decision values produced may differ, there is no protocol to tolerate $k$ failures, where $k$ is the maximum number of distinct values that may be chosen as decisions. Of particular interest was the introduction of algebraic and combinatorial topology in the study of these problems $([17,22,16,18])$. On the other hand, since in the asynchronous model the fault-tolerant consensus problem cannot be solved deterministically, solutions that have been given employ randomization or assume some form of synchrony. For a survey and for detailed references to those works cf. [11], [21].

If we want to sum these up, from the theoretical point of view, we have many surprising negative results, while the interest in the problem remains high. This is because, on one hand, it is interesting to develop a thorough understanding of the borders and relations between classes of objects with respect to their synchronization power; on the other hand, it is interesting to study more up-to-date architectures, which provide more fundamental synchronization primitives than just atomic reads and writes. Besides, it is easily noticeable that there is an important middle ground between the completely asynchronous and completely synchronous extreme; this middle ground is reasonable for modeling real concurrent systems. As a result, there is an increasing interest in the past few years in research towards defining and designing new architectures (e.g. the transactional memory [15]) or exploiting the properties of already existing ones (which are not all present in the theoretical models), in order to implement wait-free shared data objects $([13,2,6]$, to mention but a few $)$. 


\subsection{Results and comparison with previous work}

Following the direction mentioned above, in this work we consider the wait-free consensus problem in an "in-phase" multiprocessor system ([19]). In the same system model the wait-free clock synchronization problem has been earlier studied in [10]. Since by today's technology multiprocessor computers have large numbers of processors and since the probability of a crash increases with the number of processors in the system, it is vitally important to design multiprocessor systems that tolerate faults.

In an "in-phase" multiprocessor system processors share a common clock pulse; in the duration of a pulse a processor reads the shared data of one processor, does some local computation and updates its own shared data. (It should be pointed out that a processor cannot modify the contents of registers owned by other processors.) It is possible that processes in this system operate at very different speeds, i.e. miss pulses because of preemption, interrupts, page faults, or even processor crashes. So, although in a step a process atomically reads and writes, and, therefore, 2-process wait-free consensus is solvable ([14]), due to pulse misses and possible processor crashes, it is not obvious whether the $n$-process consensus problem can be solved wait-free in the system. This work presents a solution for the wait-free consensus problem with $n$ processes in an "in-phase" multiprocessor system, thus answering an open question stated in [10] and showing that this system model/architecture is strong enough to support deterministic $n$ process fault tolerant agreement. The protocol ensures that a process will reach decision within $n(n-3) / 2+3$ steps of its own in the worst case, or within $n-1$ steps when no process misses a pulse.

To the best of our knowledge no solution to this problem has been given before. Previous results that could serve as solutions can be found in $[1,4,5]$. Those protocols are for the the asynchronous model, which supports only read/write atomically, and guarantee decision in exponential, $O\left(n^{4}\right)$ and $O\left(n \log ^{2} n\right)$ expected number of steps, respectively, but at the cost of randomization. On the other hand, the $n$ process wait-free consensus protocols presented in [14] require some form of multi-writer read-modify-write or more sophisticated primitives (augmented queue, memory-to-memory-swap); the system model studied here does not provide that directly. Besides, the three protocols presented in [9] as part of a thorough analysis of the cases when consensus is solvable in message-passing systems, cannot be translated into solutions for our system model. Protocols E2 and E3 (of [9]) assume synchronous processes (no napping faults) and totally ordered messages (not just FIFO channels), respectively. Protocol E1 relies much on the nature of synchronous message communication, i.e. that a process which had a long napping failure receives all the messages sent to it during that time interval as soon as it resumes execution. In our model a process has to make $n-1$ steps to learn about shared variable modifications; during that time it might suffer a new napping fault and this might be repeated unbounded many times.

\section{The Computation Model}

The system consists of $n$ processes which are identified by distinct identity numbers, denoted by $P_{1}, \ldots, P_{n}$. The processes communicate via a set of single-writer, multi-reader atomic registers. Each one owns a subset of these registers. The owner of a register can write the 
register while all the other processes can read it. A step by a process $P_{k}$ consists of the following actions: (i) read by $P_{k}$ of the shared registers owned by some process $P_{k^{\prime}}\left(k \neq k^{\prime}\right)$, (ii) transition of $P_{k}$ 's local state (program counter, local variables), and (iii) update of its own shared registers.

We consider "in-phase" multiprocessor systems, in which all processors share a common clock pulse. Each pulse is a (possibly empty) set of process names; the set of processes that make a step in the pulse. Each process can make at most one step in one pulse; if it does not make a step in some pulse it will be said to miss that pulse. A configuration is a tuple of process states and values of the shared variables. A system execution is a sequence $c_{0} \pi_{1} c_{1} \pi_{2} \ldots$ of alternating pulses (denoted by $\pi_{x}$ ) and configurations (denoted by $c_{x}$ ); consecutive pulses are indexed with consecutive integers. Each configuration $c_{i}$ in a system execution is derived from its directly preceding configuration $c_{i-1}$ by the state transitions and the shared variable updates of the processes that make a step in pulse $\pi_{i}$; the reads of shared registers that occur in pulse $\pi_{i}$ return the respective values of $c_{i-1}$, while the updates of the shared registers in the same pulse take place in unison to derive $c_{i}$. For any pulse $\pi_{j}$ in any system execution $E$ and for any process $P_{k}$, let $\operatorname{work}\left(P_{k}, \pi_{j}\right)$ denote the number of steps that $P_{k}$ made from the beginning of $E$ (pulse $\pi_{1}$ ) until (and including) pulse $\pi_{j}$.

In the consensus problem each process $P_{k}$ is given an input value $v_{k}$ and is required to return an output value $v_{k}^{\prime}$; we call the step when this happens the return step of $P_{k}$. If $P_{k}$ makes its return step in some pulse $\pi_{j}$, it makes no more steps in subsequent pulses in the execution; if at some pulse in an execution $P_{k}$ has not made its return step yet, it is called undecided in that pulse. A wait-free consensus protocol should satisfy the following requirements for every system execution:

Wait-freedom There should be a bounded number $T$ such that: there is no process $P_{k}$ and pulse $\pi_{j}$ such that $P_{k}$ is undecided in $\pi_{j}$ and $\operatorname{work}\left(P_{k}, \pi_{j}\right)>T$.

Validity For each process $P_{k}$, its output value $v_{k}^{\prime}$ should equal the input value $v_{k^{\prime}}$ of some process $P_{k^{\prime}}$ of the system.

Consistency For any processes $P_{k}$ and $P_{k^{\prime}}$ with output values $v_{k}^{\prime}$ and $v_{k^{\prime}}^{\prime}$ it should be $v_{k}^{\prime}=v_{k^{\prime}}^{\prime}$.

\section{Description of the Protocol}

The protocol is described in C-like pseudocode in figures 1 and 2. We have adopted several conventions, like using capital case names for shared variables and capital boldface for calls to read/write shared registers. The following paragraphs describe the protocol more intuitively.

Each process $P_{k}(k \neq 1)$ first plays a game with $P_{1}$. If it wins it is called dominant (in the set $\left.\left\{P_{1}, \ldots, P_{k}\right\}\right)$ and writes that information on its $D O M_{k}$ shared register. Subsequently, the final decision is reached through stages in which partial decisions are made inductively. Let $D_{1 . . p r o c}$ denote the decision that would be taken if the system consisted only of processes $P_{1}, \ldots, P_{\text {proc }}$. The protocol tries to follow the inductive rule: $D_{1 . . n}$ is the input value of $P_{n}$, if $P_{n}$ is dominant; otherwise, $D_{1 . . n}=D_{1 . .(n-1)}$, with $D_{1 . .1}=V A L_{1}$, the input value of $P_{1}$. Each process tries to find $D_{1 . . p r o c}$ for proc $=1, \ldots, n$. After a process $P_{k}$ makes a partial decision for $D_{1 . . p r o c}$, it writes the value $v$ decided and the process identity proc on its $D E C_{k}$ 
$\operatorname{var}\left(V A L_{1}, D O M_{1}, D \_S E T_{1}, D E C_{1}\right), \ldots$,

$\left(V A L_{n}, D O M_{n}, D \_S E T_{n}, D E C_{n}\right):($ valtype, boolean, $1 . . n$, valtype) ;

${ }^{*}$ Shared var's; Initially all $=($ null $, 0,1$, null $) * /$

$\operatorname{DECIDE}(k, v a l) / *$ process $P_{k}$ with input value $v a l * /$

var $p r o c, d \_s e t, d \_s e t \_p: 1 . . n ; /^{*}$ Initially $=1,1,1 * /$

dom, dom_p, rech: boolean $; /^{*}$ Initially $=0,0,0 * /$

dec, dec_p, val_p: valtype ;

/* Local variables are globally binded in the module */

proc $R E A D \& \operatorname{check}(i) / *$ Read $P_{i}$ 's registers as first action in a step; check for decisions $* /$ begin

READ $\left(v a l_{-} p, d_{o m} p, d_{-} s e t_{-} p, d_{e} \_p\right)$ from $\left(V A L_{i}, D O M_{i}, D \_S E T_{i}, D E C_{i}\right)$;

if $d_{\_} s e t \_p \geq i$ then $d_{-} s e t:=d_{\_} s e t \_p ; d e c:=d_{e} c_{-} p ; /^{*}$ copy (partial) decision */ end

proc $U P D A T E() / *$ Update own var's as last action of step */

begin

WRITE (val,dom,d_set,dec) to $\left(V A L_{k}, D O M_{k}, D \_S E T_{k}, D E C_{k}\right)$;

end

proc recheck $(l: 1 . . n$, dec_tmp: valtype $)$

var $i: 1 . . n$;

begin

for $(i:=2 ; i<l ; i++)$ do

if $i \neq k$ do

$R E A D \& \operatorname{check}(i)$;

if $d \_s e t \geq l$ then $p r o c:=i:=d \_s e t ; / *$ advance current process ptr */

if $(i=l-1$ or $i=l-2$ and $k=l-1)$ and $d \_s e t<l$ then $d \_s e t:=l$; dec $:=$ dec_tmp ;

$U P D A T E()$;

endif

end_for

end

Figure 1: Protocol DECIDE: (a) Shared Variables and auxiliary procedures

and $D_{-} S E T_{k}$ shared registers, respectively. Let $D_{k}($ proc $)=v$ denote that mapping, which is the estimation of $P_{k}$ for $D_{1 . . p r o c} ; P_{k}$ will finally return its $D_{k}(n)$. Since processes might miss pulses and are, therefore, asynchronous, deviations from the rule for deciding $D_{1}$..proc are allowed, so that a fast process $P_{k^{\prime}}$ can meet the wait-free requirement when $P_{1}$ and $P_{\text {proc }}$ are so slow that the result of the game between them is not known at the time that $P_{k^{\prime}}$ needs to find out $D_{1 . \text {.proc }}$. The deviation is that the fast process $P_{k^{\prime}}$ (arbitrarily) considers that $P_{\text {proc }}$ is not dominant and sets $D_{k}($ proc $)=D_{k}($ proc -1$)$. Since this is done by a fast process, i.e. early enough, that information is available in the respective shared registers, for the slow 
proc $S a f e P h a s e(k) /{ }^{*}$ Estimate $D_{1 . . k}=$ decision if system consited only of $P_{1} . . P_{k} * /$

begin

$R E A D \& c h e c k($ proc $) ; /^{*}$ step $S_{1} . k:$ dominance in $\left\{P_{1}, \ldots, P_{k}\right\}$ ? (check presence of $\left.P_{1}\right) * /$ if $d_{-} s e t>1$ then $p r o c:=d \_s e t$;

else if $v_{a l} p=$ null then $d_{o m}=1$;

if $d o m$ and $k=2$ then $d \_s e t:=2 ;$ dec $:=v a l$;

$U P D A T E()$;

for $(++$ proc; proc $<k ;$ proc ++$)$ do

$R E A D \& c h e c k$ (proc) ;

if $d_{\_} s e t \geq$ proc then proc $:=d_{-}$set $; /{ }^{*}$ advance current process ptr $* /$

else if $\neg($ dom $)$ then $d_{-} s e t:=$ proc

end if ;

if $d o m_{-} p$ then $d e c:=v a l_{-} p ; /^{*}$ else $d e c=D_{k}(p r o c)=D_{k}(p r o c-1) * /$

if $p r o c=k-1$ and $d_{\_}$set $<k$ then $d_{-} s e t:=$ proc $:=k$;

end_if ;

if dom then $d e c:=v a l$;

$U P D A T E()$;

end

end_for

begin /* Main body of procedure DECIDE */

if $k=1$ then dec $:=v a l ; / *$ Step $S_{0} . k$ : announce presence */

$U P D A T E()$;

if $k \neq 1$ then $\operatorname{SafePhase}(k)$;

for $(++$ proc; proc $\leq n ;$ proc ++$)$ do

READ\&check(proc) ;

if $d_{-} s e t \geq$ proc then proc $:=d_{-} s e t ; / *$ advance current process $\mathrm{ptr} * /$

else if $d o m_{-} p$ then

if $k=1$ then $d_{-} s e t=p r o c ;$ dec $:=v a l_{-} p$;

else dec_tmp $:=v a l_{-} p ;$ rech $:=1 ; /{ }^{*}$ should recheck for "deviate" decisions */

else $d_{-} s e t=$ proc ; $/{ }^{*}$ consider proc not dominant ("deviate" decision) */

$U P D A T E()$;

end_for

if $r e c h$ then recheck $($ proc, dec_tmp); rech $:=0$;

end

return $($ dec $)$;

Figure 2: Protocol DECIDE: (b) Procedure SafePhase(k) and main body of DECIDE

processes to find out about the deviation from the rule, and, therefore, decide consistently. Naturally, each process, in each one of its steps, checks whether a final decision or a more advanced - than the one it knows so far- partial decision is reached and adopts it, thus advancing its process scanning pointer, i.e local variable proc.

The game between $P_{1}$ and an arbitrary $P_{k}$ is played as follows: Each process (including $P_{1}$ ), as its first action of the protocol - announcement or0- step- simply announces its 
participation in the game by writing its input value on its $V A L_{k}$ register. In its next step, each $P_{k}(k \neq 1)$ reads the register of $P_{1}$ and becomes dominant only if it reads that $P_{1}$ has not made its announcement step yet (i.e. if $V A L_{1}=n u l l$ ); in all other cases $P_{k}$ looses the game. $P_{1}$ plays with each $P_{\text {proc }}$ in $\left\{P_{2}, \ldots, P_{n}\right\}$, one at a step and in that order (except when advancing its local variable proc), by checking whether $P_{\text {proc }}$ is dominant; if not, $P_{\text {proc }}$ will never become dominant, since it will read that $P_{1}$ has already made its announcement step. Thus, in that case the partial decision is $D_{1 . . p r o c}=D_{1 . .(\text { proc }-1)}$ and $P_{1}$ sets $D_{1}($ proc $)=D_{1}($ proc -1$)$. Otherwise -if $P_{\text {proc }}$ is dominant-, $P_{1}$ safely (for reasons that become clear later in this section) concludes that $D_{1 . \text { proc }}=V A L_{\text {proc }}$ and sets $D_{1}($ proc $)=V A L_{\text {proc }}$.

Each process $P_{k}(k \neq 1)$ follows a protocol of 2 stages; first, using procedure SafePhase $(k)$ it estimates the partial decision $D_{1 . . k}$ and then continues for $D_{1 . .(k+1)}, \ldots, D_{1 . . n}$. After making an observation, we first describe the second phase, to give the intuition in a more clear way.

Observation 1 If a process $P_{k}$ is not dominant and reads $D O M_{k^{\prime}}=0$, then it knows that $P_{k^{\prime}}$ will not become dominant in that execution, because $P_{1}$ has made its announcement step.

Suppose that $P_{k}$ has an estimation (from procedure $S a f e P h a s e(k)$ ) of the partial decision $D_{1 . . k}$. Then, for each proc $=k+1, \ldots, n$, it estimates $D_{1 . . p r o c}$ - in that order except from advances of the local variable proc- as follows: if it reads $D O M_{\text {proc }}=0$, i.e. that $P_{\text {proc }}$ is not dominant, then, only if $P_{k}$ itself is not dominant, it can safely make a conclusion, namely that $P_{\text {proc }}$ will never become dominant (by observation 1); otherwise, it cannot conclude in a wait-free manner (i.e. without waiting for $P_{1}$ and/or $P_{\text {proc }}$ to make a step) anything about this future. Therefore, it arbitrarily considers $P_{\text {proc }}$ not dominant and writes that $D_{k}($ proc $)=D_{k}($ proc -1$)$ (by leaving its $D E C_{k}$ shared register unchanged and updating only its $D \_S E T_{k}$ into proc $)$. This implies that any other process $P_{k}^{\prime}\left(k^{\prime}<p r o c\right)$, which will possibly later read that $P_{\text {proc }}$ is dominant, before feeling free to decide $D_{k^{\prime}}($ proc $)=V A L_{\text {proc }}$, it will have to recheck for earlier, deviate decisions about $D_{1 . . p r o c}$, among processes with identity $P_{x}$, s.t. $x<$ proc (i.e. among processes that might have had to decide about that with insufficient information). Note that $P_{1}$ need not recheck for deviations in decisions for any $D_{1 . . p r o c}$ if it reads that $P_{\text {proc }}$ is dominant; if such a decision was made, this would have happened before the first step of $P_{\text {proc }}$, therefore before the 0 -step of $P_{1}$ itself, by a process $P_{x}$, s.t. $x<$ proc. Thus, $P_{1}$ would have read that decision before reading $P_{\text {proc's registers. }}$ '

Now, let's see what the first phase is. In procedure $S a f e P h a s e(k)$, if $P_{k}$ becomes dominant, in which case it should be $D_{1 . . k}=V A L_{k}$, it only has to check whether a fast process in $\left\{P_{2}, \ldots, P_{k-1}\right\}$ has earlier made a deviate decision about $D_{1 . . k}$. If $P_{k}$ is not dominant it has to estimate $D_{1 . .(k-1)}$. In both cases it suffices for $P_{k}$ to scan once the shared registers of each one of the processes in $\left\{P_{2}, \ldots, P_{k-1}\right\}$. If from a process $P_{\text {proc }}$ it reads $D O M_{\text {proc }}=0$, then it knows that $P_{\text {proc }}$ will never become dominant (by observation 1 ) and decides $D_{k}($ proc $)=$ $D_{k}($ proc -1$)$. Otherwise, if $P_{\text {proc }}$ is dominant, it decides $D_{k}($ proc $)=V A L_{\text {proc }}$. It does not have to recheck for earlier, deviate decisions for $D_{1 \text {..proc }}$, because if there was any, $P_{k}$ would have read it before reading $P_{\text {proc }}$ 's registers (by an argument similar to the one in the previous paragraph). 


\section{Correctness and Performance of the Protocol}

For what follows in this section, we need some auxiliary notation/terminology. Consider an arbitrary system execution $E$ :

- A process $P_{k}$ maps a value $v$ to the set $\{1, \ldots, p r o c\}$ in $E$ if there exists a configuration such that $D E C_{k}=v$ and $D_{-} S E T_{k}=p r o c$; we denote this mapping by $D_{k}(p r o c)$ and say that $P_{k}$ decides this value $v$ for the set $\{1, \ldots$, proc $\}$. This might happen either because $P_{k}$ copies that decision from the shared register of some other process (in $R E A D \& c h e c k$ ) or because $P_{k}$ computes that decision (in SafePhase on in the main body of $D E C I D E$ ). Note that $P_{k}$ in its return step in $E$ returns $D_{k}(n)$. In analogy with consistency for the final output value, we say that the decisions for a set $\{1, \ldots$, proc $\}$ are consistently made in $E$ by the processes of a set $P$, if for any two $P_{k}, P_{k^{\prime}} \in P$ that decide for $\{1, \ldots, p r o c\}$, it holds that $D_{k}($ proc $)=D_{k^{\prime}}($ proc $)$.

- If $s$ and $s^{\prime}$ are steps by processes, $s \rightarrow s^{\prime}$ denotes that $s$ precedes $s^{\prime}$, while $s \| \rightarrow s^{\prime}$ denotes that $s$ either precedes or is concurrent with $s^{\prime}$ in $E$; the latter is equivalent with $\neg\left(s^{\prime} \rightarrow s\right)$. The step of $P_{k}$ in which it reads $P_{\text {proc }}$ 's shared registers for the first time, not during procedure recheck, is denoted by $S_{\text {proc }} . k$. Furthermore, $S_{0} . k$ denotes the announcement, or 0 -step of $P_{k}$.

- A process $P_{k}$ is dominant in $E$, if in the configuration after $S_{1} . k$-and, henceforth in all subsequent configurations in $E$ - it is $D O M_{k}=1$.

Lemma 1 (Wait-freedom) In a system with $n$ processes, in any execution, each process $P_{k}$ makes its return step after having made at most $n(n-3) / 2+3$ steps.

Proof. (Sketch) Each process $P_{k}$ decides $D_{k}(k)$ in $k$ steps of its own; for each $D_{k}(x)$ $(k<x \leq n)$ it needs in the worst case -i.e. if recheck is necessary- $1+(x-3)$ steps of its own. Summing this all up, we have that in the worst case $P_{k}$ terminates using $D E C I D E$ in

$$
k+\sum_{x=k+1}^{n}(x-2)=n(n-3) / 2-k(k-5) / 2
$$

steps of its own. The largest value is when $k=2$ (because $P_{1}$ never rechecks and terminates in at most $n$ steps) and equals $n(n-3) / 2+3$ steps.

Lemma 2 (Validity) In each execution each process which makes a return step, outputs a value that equals the input value of some process in the execution.

Proof. (Sketch) A process $P_{k}$ that terminates returns the value $D_{k}(n)$ that decides and holds in its $D E C_{k}$ shared variable. That value came either from a copy from some process $P_{k^{\prime}}$ 's $D E C_{k^{\prime}}$ shared variable, or from an assignment to $D E C_{k}$ of the input value $V A L_{\text {proc }}$ of a process $P_{\text {proc }}$. In the latter case the lemma is straightforward; in the former case, if we trace back the origin of the value held in $D E C_{k^{\prime}}$, by the same argument, we will find that it is an input value of some process in that execution. 
Lemma 3 (Consistency) In a system with $n$ processes the decisions for all sets $\{1, \ldots, p\}$ $(1 \leq p \leq n)$ are consistently made by the processes of the set $\left\{P_{1}, \ldots, P_{n}\right\}$, in every system execution.

Proof. (Sketch) This can be proven by induction on the number $p$.

Consider an arbitrary execution $E$ in a system with $n$ processes. For $p=1$ the lemma holds because for any process $P_{\text {proc }}$ in $\left\{P_{1}, \ldots, P_{n}\right\}$, in order to decide $D_{\text {proc }} .1$ it should be $S_{1}$.proc $\rightarrow S_{0} .1$, which implies that the input value of $P_{1}$ is available in its $V A L_{1}$ shared variable; therefore, for each $P_{\text {proc }}$ that decides for $\{1, \ldots, 1\}$, it will be $D_{\text {proc }}(1)=V A L_{1}$. Assume the lemma holds for all $p \leq k$; we will show that it also holds for $p=k+1$. From the induction hypothesis we know that the decisions for $\{1, \ldots, k\}$ are consistently made by the processes of the set $\left\{P_{1}, \ldots, P_{n}\right\}$. In order to prove that the decisions for $\{1, \ldots, k+1\}$ are also consistently made by the processes of the set $\left\{P_{1}, \ldots, P_{n}\right\}$, there are two cases to be considered:

Case 1: $P_{k+1}$ is not dominant in $E$. Each $P_{\text {proc }} \in\left\{P_{1}, \ldots, P_{k}\right\} \cup\left\{P_{k+2}, \ldots, P_{n}\right\}$ that makes $S_{k+1}$.proc in $E$, reads $D O M_{k+1}=0$ and, therefore, can only possibly decide $D_{\text {proc }}(k+1)=$ $D_{\text {proc }}(k)$. Besides, $P_{k+1}$ itself will also decide $D_{k+1}(k+1)=D_{k+1}(k)$. Since the decisions for $\{1, \ldots, k\}$ are consistently made by the processes in $\left\{P_{1}, \ldots, P_{n}\right\}$, the same follows in this case for the decisions for $\{1, \ldots, k+1\}$.

Case 2: $P_{k+1}$ is dominant in $E$. There are two possibilities:

- Each $P_{\text {proc }}$ in $\left\{P_{1}, \ldots, P_{k}\right\} \cup\left\{P_{k+2}, \ldots, P_{n}\right\}$ that executes $S_{k+1}$.proc and decides for $\{1, \ldots, k+1\}$, reads $D O M_{k+1}=1$, and, therefore, sets $D_{\text {proc }}(k+1)=V A L_{k+1}$; similarly, $P_{k+1}$ will set $D_{k+1}(k+1)=V A L_{k+1}$. Therefore, in this case the decisions for $\{1, \ldots, k, k+1\}$ are consistently made by the processes in $\left\{P_{1}, \ldots, P_{n}\right\}$.

- Some $P_{\text {proc }}$ in $\left\{P_{1}, \ldots, P_{k}\right\}$, which executes $S_{k+1}$.proc and decides for $\{1, \ldots, k+1\}$, reads $D O M_{k+1}=0$. Clearly, proc $\neq 1$ and proc $\neq k+1$; otherwise, the dominance of $P_{k+1}$ would be contradicted. $P_{\text {proc }}$ computes $D_{\text {proc }}(k+1)=D_{\text {proc }}(k)$. It holds that:

$$
\begin{array}{lr}
S_{k+1} \cdot \text { proc } \| \rightarrow S_{1} \cdot(k+1) & \left(P_{k+1} \text { is dominant but } P_{\text {proc }} \text { reads } D O M_{k+1}=0\right) \\
S_{1} \cdot(k+1) \| \rightarrow S_{0} .1 & \left(P_{k+1} \text { is dominant in } E\right) \\
S_{1} \cdot(k+1) \rightarrow S_{\text {proc }} \cdot(k+1) \| \rightarrow S_{k} .(k+1) & (1<\text { proc } \leq k) \\
S_{0} .1 \rightarrow S_{\text {proc }} .1 \rightarrow S_{k+1} .1 & (0<\text { proc }<k+1)
\end{array}
$$

Since in $S_{k+1}$.proc process $P_{\text {proc }}$ computes $D_{\text {proc }}(k+1)=D_{\text {proc }}(k)$, the above imply that $P_{1}$ and $P_{k+1}$ will copy that decision from $P_{\text {proc }}$ 's shared register in their $S_{\text {proc }} .1$ and $S_{\text {proc }} .(k+1)$ steps, respectively, i.e. before the steps in which they would have to decide for $\{1, \ldots, k, k+1\}$.

For any $P_{\text {proc }}$ in $\left\{P_{1}, \ldots, P_{k}\right\}$ that executes $S_{k+1}$.proc ${ }^{\prime}$ and decides for $\{1, \ldots, k+1\}$, we have either that (a) $P_{\text {proc }}$ reads $D O M_{k+1}=0$ and, therefore, sets $D_{\text {proc }}(k+1)=$ $D_{\text {proc }^{\prime}}(k)$, or that (b) $P_{\text {proc }}$ reads $D O M_{k+1}=1$ and executes recheck. In the latter case, since $P_{\text {proc }}, P_{\text {proc }}$ read from $D O M_{k+1}$ values 0,1 , respectively, it holds that 


$$
S_{k+1} . p r o c \| \rightarrow S_{1} \cdot(k+1) \rightarrow S_{k+1} . p r o c^{\prime}
$$

Moreover, from the protocol we have that

$$
S_{k+1} \cdot \operatorname{proc}^{\prime} \rightarrow\left\{\operatorname{recheck}\left(k+1, V A L_{k+1}\right) \text { steps by } P_{\text {proc }}\right\}
$$

Since $P_{\text {proc }}$ in $S_{k+1}$.proc computes $D_{\text {proc }}(k+1)=D_{\text {proc }}(k)$, the above imply that $P_{\text {proc }}$ will copy that decision from $P_{\text {proc }}$ 's shared register during recheck.

For any $P_{\text {proc }}$ in $\left\{P_{k+2}, \ldots, P_{n}\right\}$ that executes $S_{k+1}$. proc ${ }^{\prime \prime}$ and decides for $\{1, \ldots, k+1\}$, we have the following: (a) if $P_{\text {proc }}$ is dominant in $E$, since $k+1<p r o c^{\prime \prime}$, from the protocol it follows that $P_{\text {proc }}$ may only copy $D_{\text {proc }}$ " $(k+1)$ from a process in $\left\{P_{1}, \ldots, P_{k}\right\}$. (b) if $P_{\text {proc }}$ is not dominant in $E$ and reads $D O M_{k+1}=0$, it will decide $D_{\text {proc }}(k+1)=D_{\text {proc }}(k)$; if it reads $D O M_{k+1}=1$ then we have the following about precedence relations of certain steps of these processes in $E$ :

$$
\begin{array}{lr}
S_{k+1} \cdot \text { proc } \| \rightarrow S_{1} \cdot(k+1) & \left(P_{k+1} \text { is dominant but } P_{\text {proc }} \text { reads } D O M_{x+1}=0\right) \\
S_{1} \cdot(k+1) \| \rightarrow S_{0} \cdot 1 & \left(P_{k+1} \text { is dominant in } E\right) \\
S_{0} \cdot 1 \rightarrow S_{1} \cdot\left(\text { proc }^{\prime \prime}\right) & \left(P_{\text {proc }} \text { " is not dominant in } E\right) \\
S_{1} \cdot\left(\text { proc }^{\prime \prime}\right) \rightarrow S_{\text {proc }} \cdot\left(\text { proc }^{\prime \prime}\right) \rightarrow S_{k+1} \cdot\left(\text { proc }^{\prime \prime}\right) & (\text { since } 1<p r o c<k+1)
\end{array}
$$

Since $P_{\text {proc }}$ in $S_{k+1}$. proc computes $D_{\text {proc }}(k+1)=D_{\text {proc }}(k)$, the above imply that in its $S_{\text {proc }} \cdot\left(\right.$ proc $\left.^{\prime \prime}\right)$ step, $P_{\text {proc }}$, will copy that decision into its $D E C_{\text {proc }}$.

Since the decisions for $\{1, \ldots, k\}$ are consistently made by the processes in $\left\{P_{1}, \ldots, P_{n}\right\}$, the same follows in this case for the decisions for $\{1, \ldots, k+1\}$.

Considering the requirements from a solution to the wait-free consensus problem, the previous lemmas imply the following theorem:

Theorem 1 The DECIDE protocol correctly implements a wait-free solution to the consensus problem in an in-phase multiprocessor system with $n$ processes, with $T=n(n-3) / 2+2$.

\section{Conclusions}

For the $n$-process consensus problem we have given a solution that tolerates processor crash and napping failures in an in-phase multiprocessor system, thus showing that this system model has a nice property, which is useful for fault-tolerant multiprocessor coordination and synchronization. Besides fault-tolerant consensus and clock synchronization, it is interesting to solve other problems efficiently and fault-tolerant in this system model; moreover, it would be useful to implement these algorithms. 


\section{References}

1. K. Aвrahamson On Achieving Consensus Using a Shared Memory. Proceedings of PODC 1988, pp. 291-302.

2. Juan Alemany and Edward W. Felten Performance Issues in Non-Blocking Synchronization on Shared Memory Multiprocessors. Proceedings of PODC 1992, pp. $125-134$.

3. James Aspnes, Maurice Herlihy Wait-free data structures in the Asynchronous PRAM. Proceedings of SPAA'90, 340-349.

4. James Aspnes, Maurice Herlihy Fast Randomized Consensus Using Shared Memory. Journal of Algorithms 11, pp. 441-461 (1990).

5. James Aspnes, Orli Warts Randomized Consensus in Expected $O\left(n \log ^{2} n\right)$ Operations Per Processor. Proceedings of FOCS 1992, pp. 137-146.

6. Greg Barnes A Method for Implementing Lock-Free Shared Data Structures. Proceedings of SPAA 1993, pp. 261-270.

7. Elisabeth Borowsky, Eli Gafni Generalized FLP Impossibility Results for $t$-resilient Asynchronous Computations. Proceedings of STOC 1993, pp. 91-100.

8. Soma Chaudhuri Agreement Is Harder Than Consensus: Set Consensus Problem in Totally Asynchronous System Systems. Proceedings of PODC 1990, pp. 311-324.

9. Danny Dolev, Cynthia Dwork, Larry Stockmeyer On the Minimal Synchronism Needed for Distributed Consensus. Journal of the ACM, vol. 34, No. 1, January 1987, pp. 77-97.

10. Shlomi Dolev, Jennifer L. Welch Wait-free clock synchronization. Proceedings of PODC'93, pp. 97-108.

11. M.J. Fischer The Consensus Problem in Unreliable Distributed Systems (A Brief Survey). YALEU/DCS/RR-273, June 1983.

12. Michael Fischer, Nancy Lynch and Michael Paterson Impossibility of Distributed Consensus with One Faulty Process. Journal of ACM, Vol. 32, No. 2, April 1985, pp. 374-382.

13. Maurice Herlihy A Methodology for Implementing Highly Concurrent Data Objects. Proceedings of $A C M$ PPoPP 1990, pp. 197-206.

14. Maurice Herlihy Wait-Free Synchronization. ACM Transactions on Programming Languages and Systems, Vol. 11, No. 1, January 1991, pp. 124-149.

15. Maurice Herlihy, J.E.B. Moss Transactional Memory: Architectural Support for Lock-Free Data Structures. Proceedings of ISCA 1993.

16. Maurice Herlihy and Sergio Rajsbaum Set Consensus Usibg Arbitrary Objects. Proceedings of PODC 1994, pp.324-333.

17. Maurice Herlihy and Nir Shavit The Asynchronous Computability Theorem for $t$-resilent Tasks. Proceedings of STOC 1993, pp. 111-120.

18. Maurice Herlihy and Nir Shavit The Asynchronous Computability Theorem for 
Wait-free Computation. Proceedings of STOC 1994.

19. K. Hwang Advanced Computer Architectures, Parallelism, Scalability, Programmability. McGraw-Hill, Inc. 1993.

20. Michael C. Loui and Hosame H. Abu-Amara Memory Requirements for Agreement among Unreliable Asynchronous Processes. Advances in Computing Research, Vol. 4 (1987), pp. 163-183

21. NANCY A. Lynch and IsaAC SAIAS Distributed Algorithms: Lecture Notes $M I T / L C S / R S S 16$ Research Seminar Series

22. Michael Saks and Fotios Zaharoglou Wait-Free k-set Agreement is impossible: The topology of Public Knowledge. Proceedings of STOC 1993, pp. 101-110. 